\title{
Gefitinib targets ZAP-70-expressing chronic lymphocytic leukemia cells and inhibits B-cell receptor signaling
}

\author{
RF Dielschneider ${ }^{1,2}$, W Xiao ${ }^{2}$, J-Y Yoon ${ }^{2,3}$, E Noh $^{2}$, V Banerji ${ }^{2,3,4}$, H Li $^{1}$, AJ Marshall ${ }^{1}$, JB Johnston ${ }^{2,3}$ and SB Gibson ${ }^{* 1,2,3,4}$
}

Chronic lymphocytic leukemia (CLL) can be divided into groups based on biomarkers of poor prognosis. The expression of the tyrosine kinase ZAP-70 (member of the Syk tyrosine kinase family) in CLL cells is associated with shorter overall survival in CLL patients. Currently, there is a lack of targeted therapies for patients with ZAP-70 expression in CLL cells. The tyrosine kinase inhibitor gefitinib has been shown to be effective at induce apoptosis in acute myeloid leukemia through inhibition of Syk. In this study, we sought to test the efficacy of gefitinib in primary human ZAP-70+ CLL cells. We demonstrate that gefitinib preferentially induces cell death in ZAP-70-expressing CLL cells with a median $\mathrm{IC}_{50}$ of $4.5 \mu \mathrm{M}$. In addition, gefitinib decreases the viability of ZAP-70+ Jurkat T leukemia cells but fails to affect T cells from CLL patients. Western blot analysis shows gefitinib reduces both basal and B-cell receptor (BCR)-stimulated phosphorylation of Syk/ZAP-70, ERK, and Akt in ZAP-70+ CLL cells. Moreover, gefitinib inhibits the pro-survival response from BCR stimulation and decreases pro-survival proteins such as Mcl-1. Finally, ZAP-70 expression sensitizes Raji cells to gefitinib treatment. These results demonstrate that gefitinib specifically targets ZAP-70+ CLL cells and inhibits the BCR cell survival pathway leading to apoptosis. This represents the likelihood of tyrosine kinase inhibitors being effective targeted treatments for ZAP-70+ CLL cells.

Cell Death and Disease (2014) 5, e1439; doi:10.1038/cddis.2014.391; published online 2 October 2014

The clinical course of chronic lymphocytic leukemia (CLL) is highly variable, and although some patients are treated at diagnosis, others may not require therapy for years. ${ }^{1}$ Biomarkers can help stratify these patients into indolent and aggressive disease categories. The aggressiveness of CLL is dependent on whether the leukemia cells have $60 \%$ of CLL population) or lack ( $40 \%$ of CLL population) mutations of the immunoglobulin variable region of the heavy chain $\left(I g V_{H}\right)$. Thus, patients with early-stage disease have a median survival of 8 years if they have unmutated $\lg V_{H}$ $\left(U n-\lg V_{H}\right)$ and 24 years if they have mutated $\lg V_{H}\left(\mathrm{Mu}-\lg V_{H}\right)$ disease. ${ }^{2}$ A surrogate marker for $\lg V_{H}$ mutational status is the expression of zeta-chain-associated protein 70 (ZAP-70); $\lg V_{H}$ mutated CLL cells are frequently ZAP-70 negative, whereas $l g V_{H}$ unmutated cells are more typically ZAP-70 positive. ${ }^{3}$ ZAP-70 staining in CLL is not an all-or-nothing phenomenon, and to maximize the correlation with $\lg V_{H}$ mutational status, a ZAP-70-positive case is defined as $\geq 20 \%$ of the CLL cells staining for ZAP-70. Like $\lg V_{H}$ status, overexpression of ZAP-70 in CLL cells is associated with aggressive disease; time to treatment is 2.6 years for ZAP-70+ patients compared with 8 years for ZAP-70 patients independent of Rai stage. ${ }^{3}$ Thus, ZAP-70 is a rationale target for therapy in CLL.
Although the clinical relevance of ZAP-70 in CLL is well known, its molecular function is less understood. ZAP-70 is a member of the Syk family of protein tyrosine kinases and is normally involved in signal transduction of the T-cell receptor in T cells. ZAP-70 overexpression in malignant $B$ cells, such as CLL cells, enhances the B-cell receptor (BCR) pathway. This pathway is a key mechanism for cell survival in CLL. ${ }^{4,5}$ Upon activation of the BCR, tyrosine kinase Lyn phosphorylates and activates Syk, leading to activation of downstream signaling pathways and upregulation of anti-apoptotic proteins, such as Mcl-1. CLL cells with both Un- IgV $V_{H}$ and high ZAP-70 expression show increased activation of proteins downstream of the BCR such as Akt, mitogen-activated protein kinase (MAPK), and NF-kB.,6,7 This suggests that alterations in the BCR signaling pathway through increased expression of the tyrosine kinase ZAP-70 are important in CLL disease progression.

Gefitinib is a tyrosine kinase inhibitor known for targeting the epidermal growth factor receptor (EGFR) and is used in the treatment of non-small-cell lung cancer and other cancers of epithelial origin. ${ }^{8}$ The drug is well tolerated, with rash and diarrhea being the only dose-limiting toxicities. Importantly to leukemias, it is not myelosuppressive. ${ }^{9}$ Apart from its effects on EGFR activity, gefitinib has shown activity against $>20$

\footnotetext{
${ }^{1}$ Department of Immunology, University of Manitoba, Winnipeg, MB, Canada; ${ }^{2}$ Manitoba Institute of Cell Biology, Winnipeg, MB, Canada; ${ }^{3}$ Department of Internal Medicine, University of Manitoba, Winnipeg, MB, Canada and ${ }^{4}$ Department of Biochemistry and Medical Genetics, University of Manitoba, Winnipeg, MB, Canada *Corresponding author: SB Gibson, Manitoba Institute of Cell Biology, CancerCare Manitoba, 675 McDermot Avenue, Winnipeg, MB R3E 0V9, Canada. Tel: +1 204 787 2051; Fax: +1 204787 2190; E-mail: spencer.gibson@med.umanitoba.ca

Abbreviations: 7AAD, 7-amino-actinomycin D; ALL, acute lymphocytic leukemia; AML, acute myeloid leukemia; APC, allophycocyanin; BCR, B-cell receptor; CLL, chronic lymphocytic leukemia; DMSO, dimethyl sulfoxide; EGFR, epidermal growth factor receptor; FBS, fetal bovine serum; FITC, fluorescein isothiocyanate; FosD, fostamatinib disodium; GAPDH, glyceraldehyde-3-phosphate dehydrogenase; GAK1, Cyclin G Associated Kinase; HBSS, Hanks' balanced salt solution; IC 50 , inhibitory concentration at $50 \%$; Mu- $/ g V_{H}$, mutated immunoglobulin variable region of heavy chain; MAPK, mitogen-activated protein kinase; MDS, myelodysplastic syndrome; MTT, 3-(4,5-Dimethylthiazol-2-yl)-2,5-diphenyltetrazolium-bromide; PARP, poly (ADP-ribose) polymerase; PI, propidium iodide; PMSF, phenylmethanesulfonyl fluoride; RBC, red blood cell; RIP2, receptor-interacting protein kinase 2; Un- $I g V_{H}$, unmutated immunoglobulin variable region of heavy chain; ZAP-70, zeta-chain-associated protein 70 Received 25.3.14; revised 04.7.14; accepted 06.8.14; Edited by M Diederich
} 
other kinase targets, including Lyn and Syk. ${ }^{10,11}$ Gefitinib has been shown activity in acute myeloid leukemia (AML), myelodysplastic syndrome (MDS), and acute lymphocytic leukemia (ALL), inducing both differentiation and cell death in vitro. ${ }^{12}$ These effects are associated with inhibition of Syk phosphorylation. Thus, although gefitinib is used to treat lung cancer by inhibiting EGFR, it has potential utility in the treatment of CLL patients with high expression of Syk family members that include ZAP-70.

In this study we show that gefitinib selectively induces apoptosis in ZAP-70-expressing CLL cells, both when unstimulated and BCR activated. These effects are associated in both cases with a reduction in overall tyrosine phosphorylation and specific decreases in Lyn/Lck, Syk/ ZAP-70, ERK1/2, and Akt phosphorylation. These changes produce a decreased expression of $\mathrm{Mcl}-1$ and blocked antiapoptotic signaling. Forced overexpression of ZAP-70 by lentiviral infection in the Raji B-cell line increases the sensitivity of the cells to gefitinib-induced apoptosis. However, normal T cells from CLL patients, which also express ZAP-70, are not affected by gefitinib. These results suggest that tyrosine kinase inhibitors such as gefitinib are a viable treatment option for ZAP-70+ CLL patients.

\section{Results}

Gefitinib targets ZAP-70+ CLL cells and leukemia cell lines. Given the efficacy of gefitinib in targeting Syk in AML, ${ }^{11,12}$ we investigated its efficacy in CLL cells expressing ZAP-70. CLL samples were defined as being ZAP-70+ if the number of positive cells was $\geq 20 \%$. The concentration of drug required to reduce cell viability by $50 \%\left(\mathrm{IC}_{50}\right)$ for gefitinib in ZAP-70+ and ZAP-70 - CLL cells was assessed using the MTT (3,3-(4,5-Dimethylthiazol-2-yl)-2,5-diphenyltetrazolium bromide) cell viability assay. The median gefitinb $I_{50}$ for ZAP-70+ CLL cells was $4.5 \mu \mathrm{M}$ and $>15 \mu \mathrm{M}$ for ZAP-70 cells that was statistically significant (Table 1 and Figure 1a). Overall, $77 \%$ of ZAP-70+ patient cells and $30 \%$ of ZAP-70 patients responded to gefitinib treatment as defined by the median $\mathrm{IC}_{50}$ concentration. In addition, there did not appear to be cross-resistance to fludarabine in the ZAP-70+ samples, and most fludarabine-resistant cases were sensitive to gefitinib (Supplementary Table 1).

Interestingly, another EGFR tyrosine kinase inhibitor, erlotinib, had no activity against the ZAP-70+ CLL cells. There was also no significant difference between the median $I_{50}$ of fludarabine in ZAP-70+ and ZAP-70 - CLL cells $(5.4 \mu \mathrm{M}$ compared with $7.0 \mu \mathrm{M})$. There was also no significant difference in the median $I_{50}$ values of gefitinib when all cases were stratified by $\lg V_{H}\left(7.0 \mu \mathrm{M}\right.$ for Un- $\lg V_{H}$ compared with $8.3 \mu \mathrm{M}$ or $\mathrm{Mu}-\lg V_{H}$ ) or if ZAP-70+ cases were stratified by mutational status $\left(4.0 \mu \mathrm{M}\right.$ for ZAP-70+/Mu-lg $V_{H}$ compared with $6.0 \mu \mathrm{M}$ for ZAP-70+/Un-lg $\left.V_{H}\right)$.

To confirm the MTT results, primary CLL cells were treated in vitro with gefitinib and cell death was analyzed by flow cytometry after $24 \mathrm{~h}$. Although the median $\mathrm{IC}_{50}$ was $4.5 \mu \mathrm{M}$ by MTT assay $72 \mathrm{~h}$ post treatment, a higher dose of $10 \mu \mathrm{M}$ was chosen for cell death experiments because of the shorter time frame of $24 \mathrm{~h}$ to detect cell death. Gefitinib treatment increased apoptosis in ZAP-70+ primary CLL cells as detected by an increase in annexin V-stained cells. After $24 \mathrm{~h}$, the number of annexin $\mathrm{V}$-positive CLL cells ranged from 25 to $85 \%$ (Figure 1b). After $72 \mathrm{~h}$, cell death was seen at doses as low as $1 \mu \mathrm{M}$ and increased in a dose-dependent manner (Figure 1c). Cell death was accompanied by increased cleavage of poly (ADP-ribose) polymerase (PARP) and caspase 3 and decreased Mcl-1 expression (Figure 1d and Supplementary Figure 1).

To test whether gefitinib could be give a synergistic apoptotic response with standard chemotherapeutic agents used to treat CLL, gefitinib was added alone or in combination with fludarabine. The combined effect of the two drugs failed to give a synergistic apoptotic response (Supplementary Figure 2a).

Based on standard definitions, ZAP-70+ CLL samples were defined as having $\geq 20 \%$ of their leukemia cells expressing ZAP- $70,{ }^{3}$ but changing the threshold of positive cells did not influence the results. To determine whether gefitinib was targeting only the ZAP-70+ cells or was affecting all cell populations, the viability of both ZAP-70+ and ZAP-70 - cells was measured within the same sample. Gefitinib decreased the percentage of viable annexin V-ZAP-70+ CLL cells compared with dimethyl sulfoxide (DMSO)-treated controls in six different CLL samples (Supplementary Figure 2b). This indicates gefitinib is selectively inducing apoptosis in ZAP-70+ CLL cells. This preference for ZAP-70+ CLL cells cannot be from another gefitinib target, as CLL cells do not express EGFR and do not differentially express receptor-interacting protein kinase 2 (RIP2) or cyclin G-associated kinase 1 (GAK1; Supplementary Figure 3).

To support the role that ZAP-70 expression plays in gefitinibinduced apoptosis, we examined four leukemia/lymphoma cell lines for their sensitivity to gefitinib treatment. The $B$ cellderived NALM6, BJAB, and I-83 cell lines do not express ZAP-70, whereas the $\mathrm{T}$ cell-derived Jurkat cell line does

Table 1 Gefitinib, erlotinib, and fludarabine $I_{50}$ values determined by MTT assays in ZAP-70+, ZAP-70 -, mutated $l g V_{H}$, and unmutated $I g V_{H}$ primary CLL cells treated for $24 \mathrm{~h}$

\begin{tabular}{|c|c|c|c|}
\hline CLL patients & Gefitinib median $I_{50}$ & Erlotinib median IC $_{50}$ & Fludarabine median $I_{50}$ \\
\hline $\begin{array}{l}\text { ZAP-70+ } \\
\text { ZAP-70- } \\
\text { Mutated (Mu-) } \lg V_{H} \\
\text { Unmutated (Un-) } \lg V_{H} \\
\text { ZAP-70+ and Mu-lgV } \\
\text { ZAP-70+ and Un- } \lg V_{H}\end{array}$ & $\begin{array}{c}4.5 \mu \mathrm{M}(n=22)^{\mathrm{a}} \\
>15.0 \mu \mathrm{M}(n=23) \\
\text { 7.0 } \mu \mathrm{M}(n=26) \\
8.3 \mu \mathrm{M}(n=13) \\
4.0 \mu \mathrm{M}(n=9) \\
6.0 \mu \mathrm{M}(n=11)\end{array}$ & $\begin{array}{l}>40.0 \mu \mathrm{M}(n=8) \\
>40.0 \mu \mathrm{M}(n=10) \\
>40.0 \mu \mathrm{M}(n=12) \\
>40.0 \mu \mathrm{M}(n=5) \\
>40.0 \mu \mathrm{M}(n=2) \\
>40.0 \mu \mathrm{M}(n=5)\end{array}$ & $\begin{array}{c}5.4 \mu \mathrm{M}(n=14) \\
7.0 \mu \mathrm{M}(n=16) \\
7.0 \mu \mathrm{M}(n=23) \\
5.4 \mu \mathrm{M}(n=10) \\
13.2 \mu \mathrm{M}(n=6) \\
5.4 \mu \mathrm{M}(n=8)\end{array}$ \\
\hline
\end{tabular}

a Statistical significance of gefitinib median $\mathrm{IC}_{50}(P<0.05)$ between ZAP-70+ and ZAP-70 - cells. 

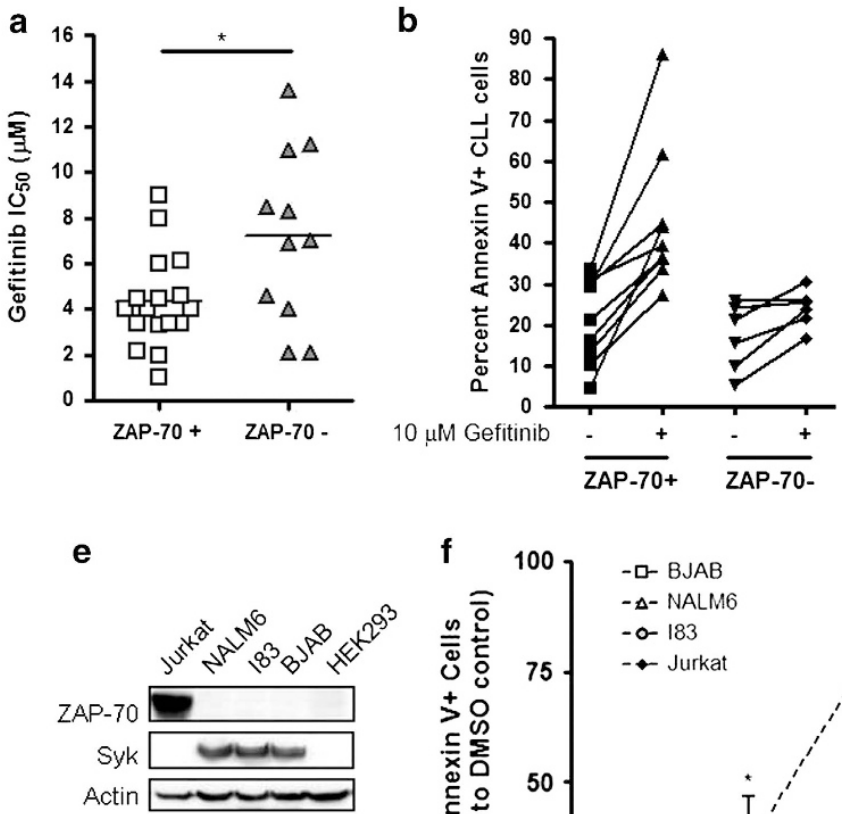

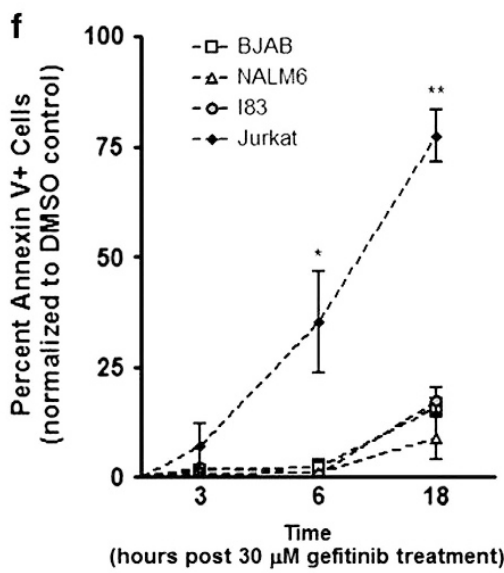

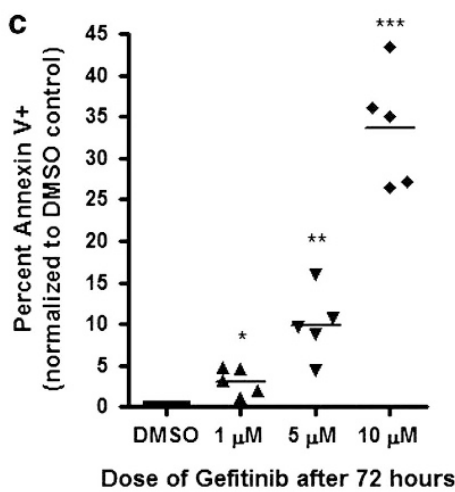
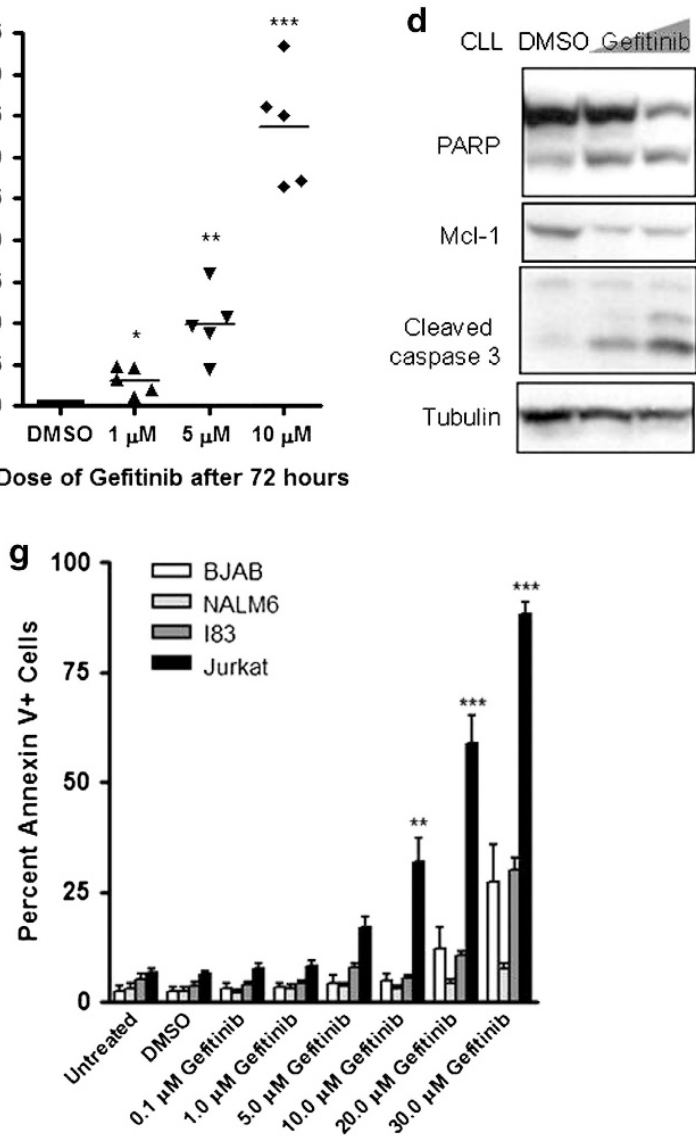

Figure 1 Gefitinib targets ZAP-70+ CLL cells. (a) Distribution of gefitinib IC ${ }_{50}$ values in ZAP-70+ $(n=22$ tested) or ZAP-70 - $(n=23$ tested) patient samples after $24 \mathrm{~h}$ of treatment. Only values within tested range of $1-15 \mu \mathrm{M}$ are shown; values outside tested range were excluded. (b) Percentage of annexin V+ CLL cells from ZAP-70+ or ZAP-70 - patients treated for $24 \mathrm{~h}$ with $10 \mu \mathrm{M}$ gefitinib. (c) Percentage of annexin $\mathrm{V}+\mathrm{CLL}$ cells from 5 different ZAP-70+ patients after $72 \mathrm{~h}$ of treatment with DMSO, and 1, 5, or $10 \mu \mathrm{M}$ gefitinib. (d) Lysates were collected after $24 \mathrm{~h}$ of CLL cells treated with DMSO, $10 \mu \mathrm{M}$ gefitinib, or $15 \mu \mathrm{M}$ gefitinib. (e) Western blot with lysates of ZAP-70 - and ZAP-70+ lymphoid cell lines compared with ZAP-70 - and Syk - HEK293 cell lines. (f) Treatment of lymphoid cell lines with $30 \mu \mathrm{M}$ gefitinib for 3, 6, and 18 h normalized to DMSO-treated control. Three independent experiments are shown with standard errors (paired tt-est). (g) Lymphoid cell lines treated with 0.1 to $30.0 \mu \mathrm{M}$ gefitinib for $24 \mathrm{~h}$. Cell death analyzed by staining with Annexin V-FITC and 7AAD and analysis by flow cytometry. Three independent experiments are shown with standard errors (paired $t$-test). ${ }^{*} P<0.05,{ }^{* \star} P<0.01$, or ${ }^{* * *} P<0.001$

express high levels of ZAP-70. Conversely, the B-cell lines expressed Syk, whereas Jurkat cells do not (Figure 1e). Human epithelial kidney cell line HEK293 was used as a negative control for both Syk and ZAP-70 expression. Gefitinib treatment over an 18-h time course revealed that only Jurkat cells showed significant apoptosis as detected by annexin $\mathrm{V}$ staining assay (Figure 1f). When the cell lines were treated with a range of gefitinib concentrations $(0.1$ to $30 \mu \mathrm{M})$ only Jurkat cells showed a significant increase in apoptosis; there were $80 \%$ annexin $\mathrm{V}_{+}$Jurkat cells but $<40 \%$ annexin $\mathrm{V}_{+}$ B-cell lines after treatment of $30 \mu \mathrm{M}$ gefitinib for $18-24 \mathrm{~h}$ (Figures $1 \mathrm{f}$ and $\mathrm{g}$ ). The Src tyrosine kinase inhibitor dasatinib did not show this bias toward Jurkat cells (Supplementary Figure 4), but this cannot rule out differential internalization of gefitinib in different cell lines.

As gefitinib had a significant effect against the Jurkat leukemia T-cell line, we determined whether normal $\mathrm{T}$ cells were also sensitive to gefitinib. We isolated mononuclear cells from peripheral blood of ZAP-70+ patients with lymphocyte count $<40 \times 10^{9}$ cells/l and treated the cells with $10 \mu \mathrm{M}$ gefitinib. This dose was chosen to agree with gefitinib treatments on CLL cells. The T and B cells were kept at the ratio observed in the peripheral blood. The CLL cells showed apoptosis following treatment with gefitinib, whereas the $\mathrm{T}$ cells were resistant (Figures $2 \mathrm{a}$ and $\mathrm{b}$ ). Even after $72 \mathrm{~h}$ of treatment with $10 \mu \mathrm{M}$ gefitinib, only ZAP-70+ CLL cells responded to treatment (Supplementary Figure 5), suggesting that gefitinib only targets malignant leukemia cells expressing ZAP-70.

Gefitinib inhibits basal and BCR signaling, preventing downstream ERK and Akt activation. In cell lines, gefitinib treatment reduced tyrosine phosphorylation of ZAP-70 in a dose-dependent manner in CD3-stimulated Jurkat T cells, but not Syk in BCR-stimulated BJAB B cells. There was no decrease in Lyn or Lck phosphorylation in BJAB or Jurkat cells, respectively (Figure 3 ). 
a

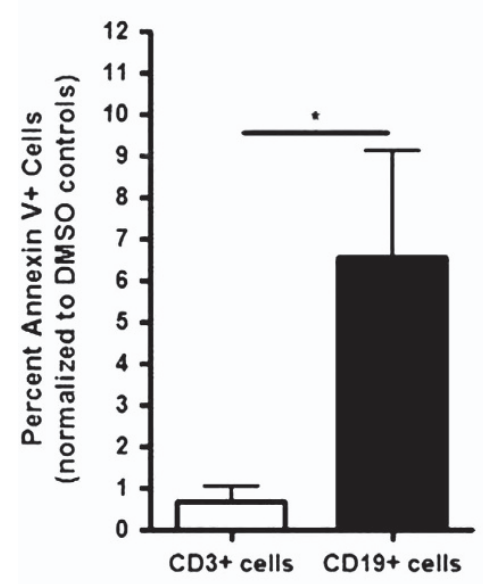

b
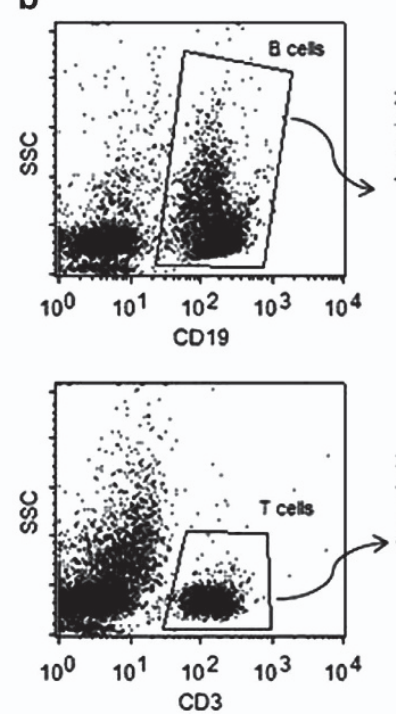
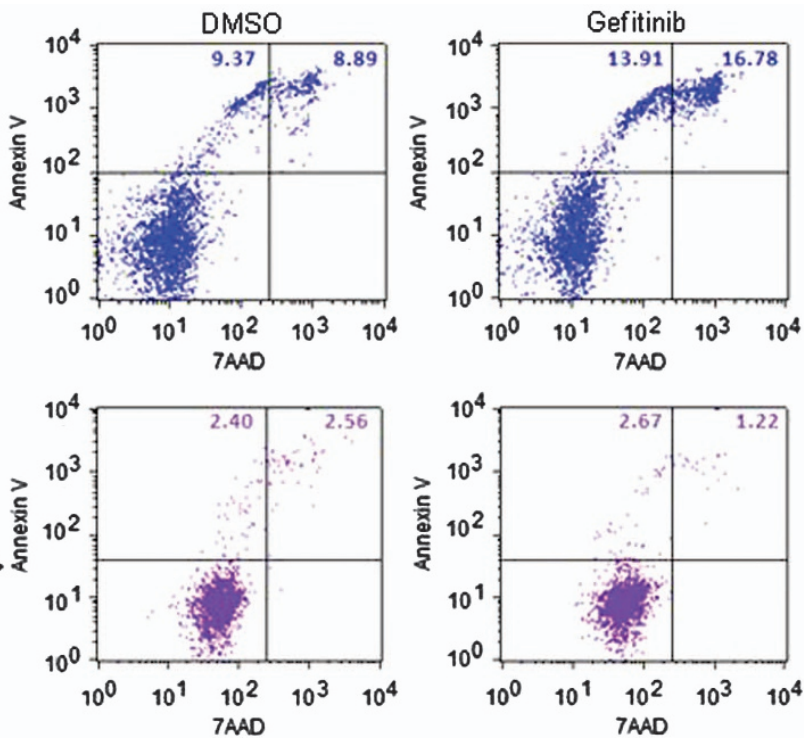

Figure 2 Gefitinib targets ZAP-70+ CLL cells but not ZAP-70+ T cells. (a) Percent Annexin V+ T cells and CLL cells from 4 different patients treated with $10 \mu \mathrm{M}$ gefitinib for $24 \mathrm{~h}$ as analyzed by flow cytometry. Graphed with standard errors and analyzed by Mann-Whitney test. (b) Flow cytometry plots of Annexin V-FITC and 7AAD+ CLL cells (gated as CD19-APC+ cells) or T cells (gated as CD3-PE+). ${ }^{*} P<0.05$
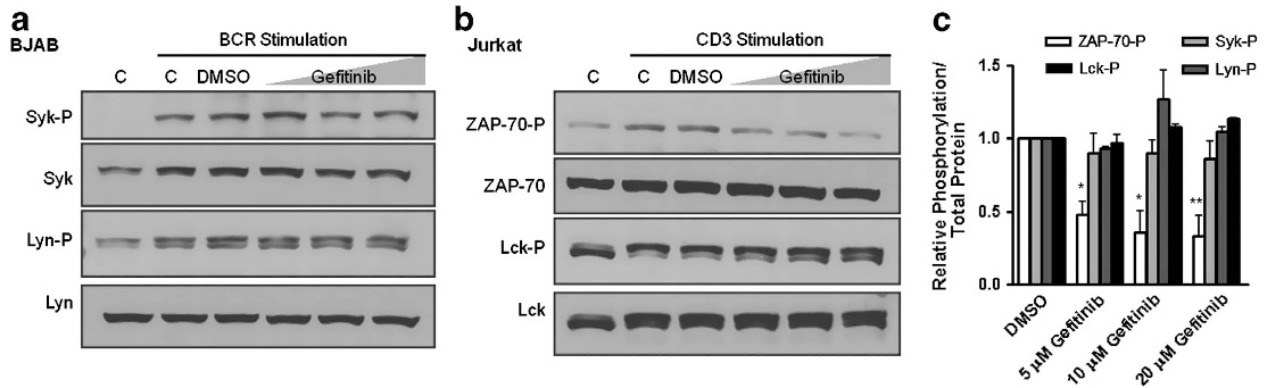

Figure 3 Gefitinib decreases ZAP-70 phosphorylation in Jurkat cells. (a) BJAB or (b) Jurkat cells treated with DMSO, and 5, 10, and $20 \mu \mathrm{M}$ gefitinib, and stimulated with anti-lgM or anti-CD3, respectively. Untreated control cells (C) were left unstimulated (lane 1) or stimulated (lane 2). Lysates collected after $1 \mathrm{~h}$ and analyzed by western blot for Syk/ZAP-70 and Lyn/Lck phosphorylation. (c) Bands quantified using ImageJ of three independent experiments. Standard errors are shown. ${ }^{*} P<0.05,{ }^{* *} P<0.01$

In primary CLL cells, we found gefitinib treatment reduced total cellular tyrosine phosphorylation, both from the basal level and from the BCR-stimulated level, in ZAP-70+ CLL cells over a range of gefitinib concentrations and incubation times (Figures $4 a-c$ ). However, this was not observed in ZAP-70 CLL cells. The decrease in overall cellular tyrosine phosphorylation was evident after $1 \mathrm{~h}$, and decreased further after $24 \mathrm{~h}$ (Figures $4 \mathrm{a}-\mathrm{C}$ ). Tyrosine phosphorylation did not decrease in ZAP-70 - CLL cells treated with increasing doses of gefitinib (Supplementary Figure 6a). However, even at doses as low as $1 \mu \mathrm{M}$, gefitinib decreased tyrosine phosphorylation in ZAP-70+ CLL cells (Supplementary Figure 6c). As a control, erlotinib failed to decrease tyrosine phosphorylation in primary CLL cells (data not shown). Unlike cell lines, there were specific decreases in both Syk/ZAP-70 and Lyn/Lck phosphorylation in ZAP-70+ CLL cells after $1 \mathrm{~h}$ of gefitinib treatment (Figure 4d). Quantification of these decreases showed that Syk/ZAP-70 phosphorylation appeared to decrease slightly more than Lyn/Lck phosphorylation after gefitinib treatment (Figure 4e).
Because of the fact that phospho-antibodies recognized both Syk and ZAP-70 phosphorylation, we performed immunoprecipitation using specific Syk and ZAP-70 antibodies and western blotting for phospho-tyrosine after BCR stimulation. Each approach showed that both Syk and ZAP-70 phosphorylation decreased following gefitinib treatment (Figure $4 \mathrm{f}$ and Supplementary Figure 6d). Immunoprecipitation was also performed with phospho-tyrosine antibodies and then blotted first for ZAP-70 and then for Syk (data not shown).

The activity of gefitinib was compared with ibrutinib and dasatinib, the two tyrosine kinase inhibitors under clinical investigation in CLL. The downstream effect of gefitinib was the same as these other two drugs and all three prevented phosphorylation of Akt and ERK after BCR stimulation (Figures $4 \mathrm{~g}$ and $\mathrm{h}$ ). Even when the primary CLL cells had high basal Akt phosphorylation, which did not increase with BCR stimulation, there was still an inhibition of phosphorylation with the tyrosine kinase inhibitors (data not shown). This inhibition was not observed with fludarabine that was used as a negative control. BCR stimulation alone served as a positive control. 
a

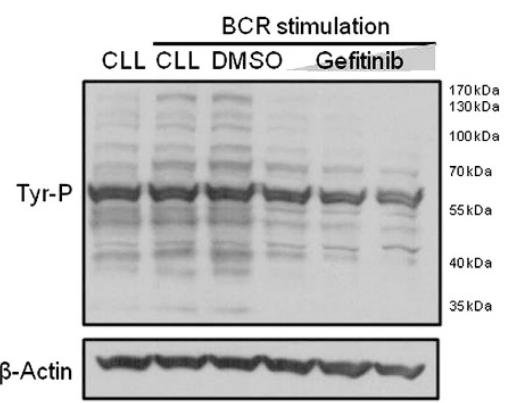

d

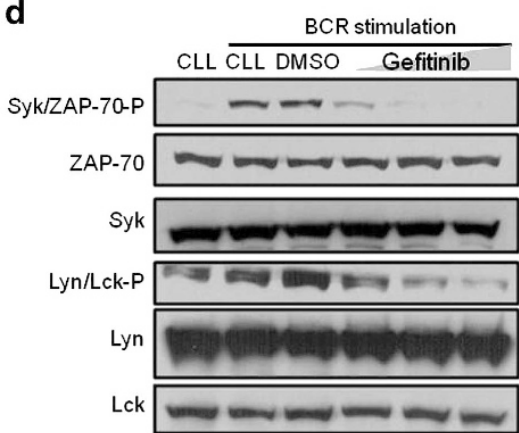

g

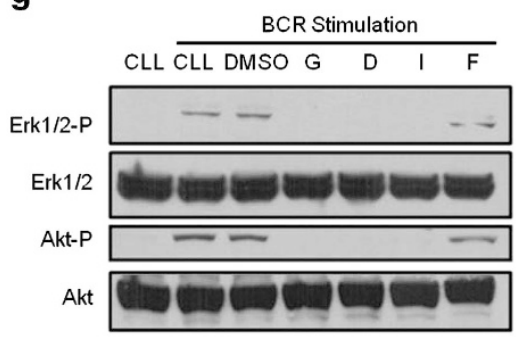

b

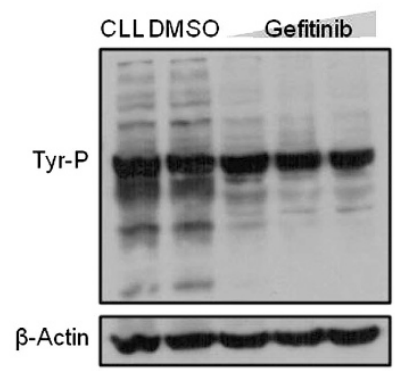

e

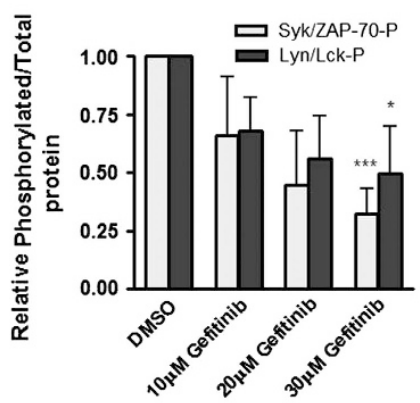

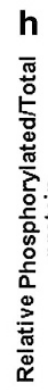

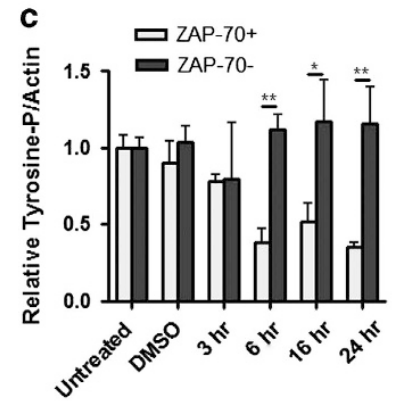

f

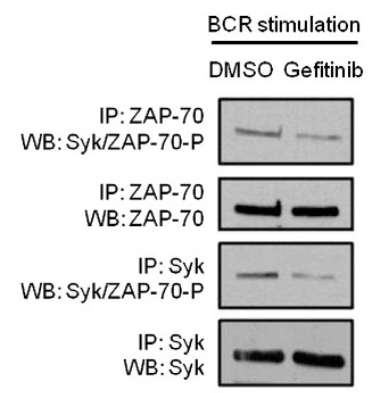

i

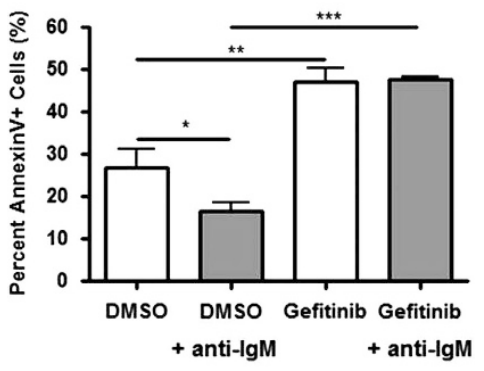

Figure 4 Gefitinib targets BCR pathway and inhibits survival response. Primary ZAP-70+ CLL cells were treated with DMSO, and 10, 20, or 30 $\mu$ M gefitinib, and stimulated with anti-IgM (a) or left unstimulated (b). Lysates were collected after $1 \mathrm{~h}$ and analyzed for tyrosine phosphorylation by western blot. The experiment was repeated with three different patient samples. (c) Lysates of a ZAP-70+ and a ZAP-70 - CLL patient sample treated with $10 \mu \mathrm{M}$ gefitinib for 3, 6, 16, and $24 \mathrm{~h}$ analyzed by slot blot for tyrosine phosphorylation (unpaired $t$-test). (d) ZAP-70+ CLL cells were treated with DMSO, and 10, 20, or $30 \mu \mathrm{M}$ gefitinib, and stimulated with anti-IgM. Lysates were collected after $1 \mathrm{~h}$ and analyzed for ZAP-70/Syk and Lyn/Lck phosphorylation by western blot. The experiment was repeated with four different ZAP-70+ CLL samples, all of which were compared using densitometry (e) calculated using ImageJ. Densitometry values of phosphorylated Syk/ZAP-70 or Lyn/Lck first normalized to DMSO, and then to total protein. (f) Immunoprecipitation of ZAP-70 and Syk from a primary CLL patient sample and analyzed for tyrosine phosphorylation by western blot after $1 \mathrm{~h}$ of $30 \mu \mathrm{M}$ gefitinib treatment. Representative of $n=3$ is quantified in Supplementary Figure 6. (g) ZAP-70+ CLL cells were treated with DMSO, $30 \mu \mathrm{M}$ gefitinib (lane G), $10 \mu \mathrm{M}$ dasatinib (lane D), $10 \mu \mathrm{M}$ ibrutinib (lane I), or $10 \mu \mathrm{M}$ fludarabine (lane F) and stimulated with anti-lgM. The experiment was repeated in two different ZAP-70+ CLL samples and compared using densitometry (h). Lysates were collected after $1 \mathrm{~h}$ and analyzed for ERK1/2 and Akt phosphorylation by western blot. The experiment was repeated with two different patient samples. (i) ZAP-70+ CLL sample was pretreated with $10 \mu \mathrm{M}$ gefitinib for $30 \mathrm{~min}$, stimulated with plate-immobilized anti-lgM for 30 min, and cultured for $24 \mathrm{~h}$. Samples were stained with Annexin V-FITC and analyzed by flow cytometry (unpaired $t$-test). Single representative experiment done in triplicate with S.D. is shown. The experiment repeated with three different patient samples. ${ }^{\star} P<0.05$, ${ }^{\star \star} P<0.01$, or ${ }^{\star \star \star} P<0.001$

As signaling through the BCR promotes cell survival, we determined whether inhibition of this signaling pathway by gefitinib decreased survival. Primary CLL cells were treated with $10 \mu \mathrm{M}$ gefitinib, BCR activated for $30 \mathrm{~min}$ by immobilized anti-lgM, and cell death quantitated by annexin- $V$ staining after $24 \mathrm{~h}$. We found that anti-IgM protected CLL cells from spontaneous apoptosis but failed to protect CLL cells from gefitinib treatment (Figure 4i).

Forced overexpression of ZAP-70 increases sensitivity to gefitinib. To determine whether ZAP-70 plays a role in the susceptibility of a patient to gefitinib, we tested the response of the ZAP-70-negative lymphoma-derived $B$-cell line Raji transduced with GFP-expressing vector or ZAP-70-expressing vector. Expression of ZAP-70 was confirmed by western blot (Figure 5a) and flow cytometry and expression of GFP was confirmed by flow cytometry (Supplementary Figure 7). We found that gefitinib treatment lowered Syk/ZAP-70 phosphorylation but not Lyn phosphorylation in ZAP-70-expressing Raji cells (Figure 5b). Western blot analysis showed constitutive phosphorylation of Syk/ ZAP-70 in Raji cells overexpressing ZAP-70 (Figure 5b), 

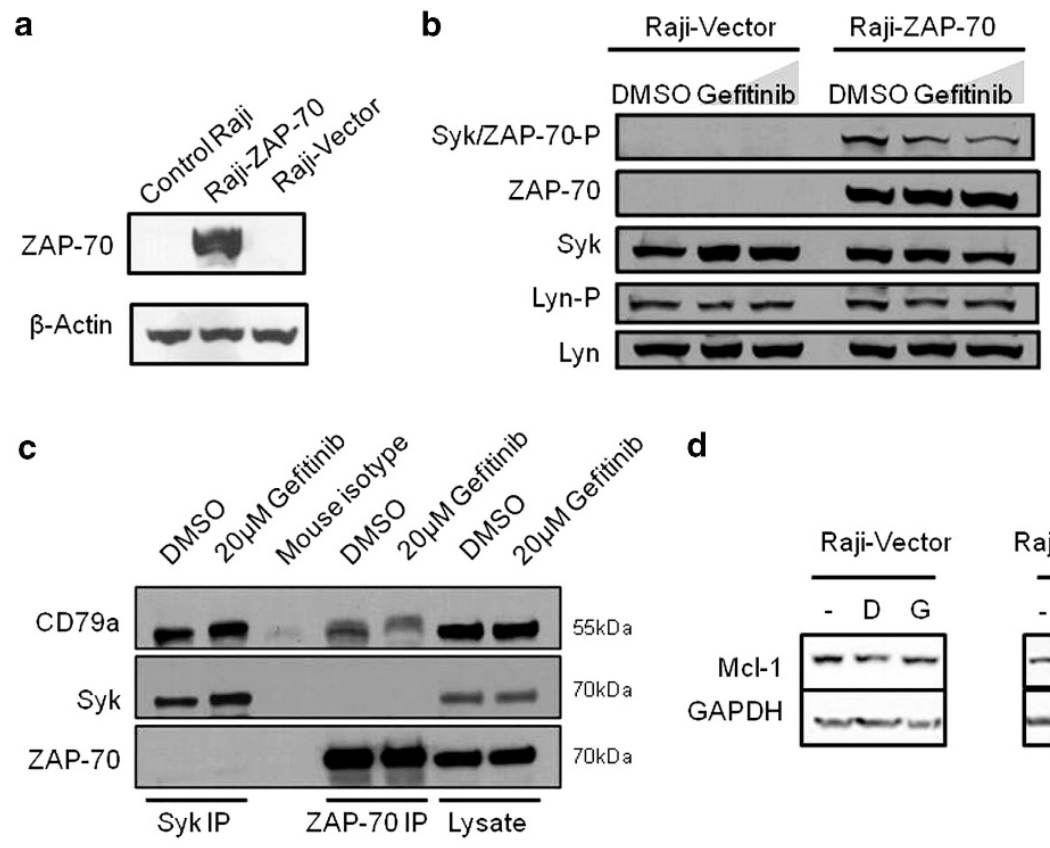

d

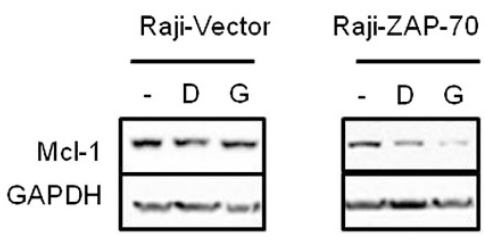

e

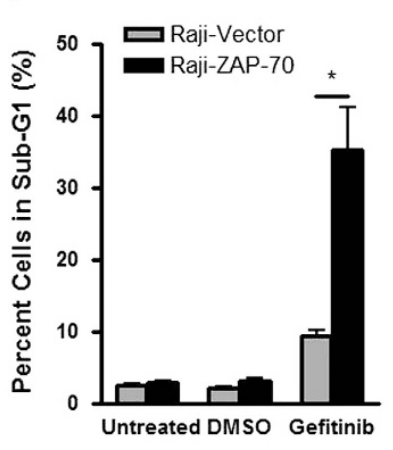

$\mathbf{f}$

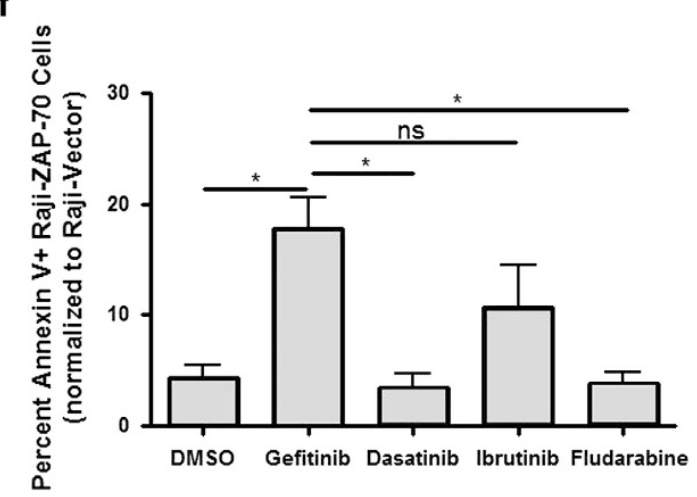

Figure 5 ZAP-70 expression sensitizes Raji cells to gefitinib treatment. Raji cells transduced with GFP control vector or ZAP-70 vector (a) were treated with DMSO, and 20 or $30 \mu \mathrm{M}$ gefitinib, or left untreated for $1 \mathrm{~h}$ and analyzed for Syk/ZAP-70 phosphorylation by western blot (b). (c) Co-immunoprecipitation of Syk or ZAP-70 with CD79a in RajiZAP-70 cells treated with DMSO or $20 \mu \mathrm{M}$ gefitinib for $24 \mathrm{~h}$ and then lysed in $0.2 \%$ CHAPS buffer. (d) Lysates of untreated (-) and treated with DMSO (D) or gefitinib (G) RajiZAP-70 and Vector cells were analyzed by western blot for Mcl-1 degradation. (e) Raji-vector and Raji-ZAP-70 cells were treated with $20 \mu \mathrm{M}$ gefitinib and stained with propidium iodide and analyzed by flow cytometry. Results from three independent experiments are shown (unpaired $t$-test). (f) Raji-vector and Raji-ZAP-70 cells were treated with DMSO, $20 \mu \mathrm{M}$ gefitinib, $30 \mu \mathrm{M}$ dasatinib, $20 \mu \mathrm{M}$ ibrutinib, or $20 \mu \mathrm{M}$ fludarabine for $24 \mathrm{~h}$ and then stained with Annexin V-APC and 7-AAD and analyzed by flow cytometry. Cell death of Raji-ZAP-70 cells normalized to Raji-vector cells. Results from three independent experiments are shown. ${ }^{*} P<0.05$

which is congruent with previously published literature. ${ }^{13}$ Upon BCR activation, Syk/ZAP-70 binds to the tyrosine phosphorylated co-receptor CD79a. We found that there was no difference in Syk or ZAP-70 binding to CD79a after gefitinib treatment (Figure $5 \mathrm{c}$ ). This suggests that CD79a is still tyrosine phosphorylated after gefitinib treatment.

We next investigated the sensitivity of gefitinib in ZAP-70overexpressing Raji cells. Raji cells overexpressing ZAP-70 had increased sensitivity to gefitinib compared with cells with vector alone as measured by greater degradation of $\mathrm{Mcl}-1$ (Figure $5 d$ ), greater DNA fragmentation as measured by subG1 peak analysis (Figure $5 e$ ), and greater annexin $\mathrm{V}$ and 7-amino-actinomycin D (7AAD) staining (Figure 5f). These results were confirmed with different cell passages, and different cell death staining techniques (PI staining and annexin V staining). We further knocked down ZAP-70 in Raji cells and showed reduced Erk phosphorylation and Mcl-1 expression, indicating changes in downstream targets of gefitinib were due to ZAP-70 overexpression (Supplementary Figure 8). In addition, gefitinib treatment was compared with dasatinib and ibrutinib. Gefitinib had the greatest effect of all the drugs on Raji cells overexpressing ZAP-70, as compared with Raji cells with vector alone (Figure $5 f$ ). Dasatinib had little effect on Raji cells, whether they overexpressed ZAP-70 or not, only increasing cell death by $3-10 \%$. Ibrutinib had a greater effect on ZAP-70-overexpressing Raji cells, as compared with the non-ZAP-70-expressing cells, but there was less cell death than seen with gefitinib using this same drug dosage $(20 \mu \mathrm{M})$. This increased sensitivity of the ZAP-70expressing Raji cells was not seen with fludarabine treatment, and this was used as a negative control (Figure 5f). 
Unfortunately, the complementary experiments to treat Jurkat cells with ZAP-70 knockdown were not feasible because knockdown of ZAP-70 led to increased cell death compared with control siRNA (Supplementary Figure 9).

\section{Discussion}

In this study the tyrosine kinase inhibitor gefitinib, originally used to inhibit EGFR kinase activation in lung cancer, is also cytotoxic to primary CLL cells that overexpress ZAP-70. When these cells undergo BCR activation, gefitinib can inhibit phosphorylation of Lyn/Lck, Syk/ZAP-70, ERK1/2, and Akt within $1 \mathrm{~h}$. These results are similar to those seen with $5-10 \mu \mathrm{M}$ gefitinib in AML and MDS cells, ${ }^{11}$ where gefitinib functions through an EGFR-independent mechanism targeting Syk activation. Using the MTT assay, which we and others have shown to be predictive of clinical response to fludarabine and chlorambucil, ${ }^{14}$ the median $\mathrm{IC}_{50}$ of gefitinib was $4.5 \mu \mathrm{M}$ in ZAP-70+ CLL cells but $>15 \mu \mathrm{M}$ in ZAP-70 - cells.

There has been considerable interest in the evaluation of tyrosine kinase inhibitors for the treatment of CLL. Dasatinib is normally used to treat chronic myeloid leukemia and is a tyrosine kinase that inhibits the Src family member Abl. ${ }^{15}$ However, it is also cytotoxic to CLL cells, including ZAP-70+ cells, and it has been suggested that dasatinib is targeting tyrosine kinase Lyn. ${ }^{15}$ In addition, Syk has been targeted in CLL with the tyrosine kinase inhibitor, R406 (fostamatinib). The effect of R406 was greatest in cells with high levels of Syk that were Un- IgV $V_{H}$ and expressed ZAP-70. ${ }^{16}$ However, R406 had no effect on the phosphorylation of other tyrosine kinases, such as ZAP-70. ${ }^{16}$ Recent evidence has indicated that these findings are clinically relevant as the pro-drug for R406, fostamatinib disodium (FosD), is clinically active in CLL patients. ${ }^{17}$ Two novel Syk inhibitors, PRT318 and P505-15, have recently been shown to suppress CLL activation and migration in vitro. ${ }^{18}$ Besides these tyrosine kinase inhibitors, the Bruton's tyrosine kinase inhibitor, ibrutinib, has shown potent activity in CLL, both in vitro and in vivo, ${ }^{19,20}$ and the multikinase inhibitor, sorafenib, has also been shown to induce apoptosis in CLL cells in vitro. ${ }^{6}$ These inhibitors were active against both ZAP-70- and ZAP-70+ CLL cells, whereas gefitinib was the only tyrosine kinase inhibitor shown to selectively sensitize ZAP-70+ CLL cells to undergo apoptosis. The long-term toxicity and efficacy of these tyrosine kinase inhibitors in CLL is unknown and it will be years before they become FDA approved for use in this disease. In contrast, gefitinib is already approved by the FDA for the treatment of lung cancer and has been used for many years in the clinic. It has the potential advantage in CLL in being specifically cytotoxic to ZAP-70+ CLL cells, and not being myelo- or immuno-suppressive.

Gefitinib has recently been shown to accumulate in solid tumors. Haura et al. ${ }^{21}$ found $22 \mu \mathrm{M}$ in lung tumor, and McKillop et al. ${ }^{22}$ found $16.7 \mu \mathrm{M}$ in breast tumor. These doses were 40 and 42 times higher than the concentration observed in the plasma, respectively. We predict that this accumulation of gefitinib at the cancer site would also occur in leukemia patients. Therefore, low plasma concentrations from patients with solid tumors may not be appropriate values to consider when testing doses of gefitinib on leukemic cells that reside in the blood and lymphoid tissues. In addition, in vitro experiments cannot recapitulate the dosing scheme that would be used in vivo. Gefitinib cytotoxic concentrations in ZAP-70+ CLL cells were similar to its concentrations that induce apoptosis in AML cells. In the future, we will focus on in vivo models testing gefitinib in various drug combinations for effectiveness.

The blood and lymphatic systems consist of distinct microenvironments that include blood, bone marrow, spleen, and lymph nodes. As cells traffic through these microenvironments, dynamic cell-cell interactions occur between mobile cells and tissue-resident cells. ZAP-70+ CLL cells tend to localize to the nodes and this is associated with more aggressive disease. ${ }^{3}$ One of the most important signals from the microenvironment for cell survival is BCR activation. ${ }^{5,23,24}$ Upon activation of the BCR, the tyrosine kinase Lyn phosphorylates and activates Syk, leading to activation of downstream signaling pathways such as Akt, MAPK, and NF$\kappa \mathrm{B}$, upregulation of anti-apoptotic proteins such as $\mathrm{Mcl}-1$, and inactivation of pro-apoptotic protein BIM. These changes lead to increased cell survival. ${ }^{24,25} \mathrm{CLL}$ cells with both Un- $I g V_{H}$ and high ZAP-70 expression show increased BCR signaling. ${ }^{24,25}$ This suggests that alterations in the BCR signaling pathway are important in CLL disease progression. In the present study, we showed that gefitinib blocked both ERK and Akt activation leading to a decrease in Mcl-1 expression and apoptosis. This mechanism of cell death may be common among the tyrosine kinase inhibitors. ${ }^{26}$ The evidence that ZAP-70 expression sensitizes cells to gefitinib and that gefitinib targets the BCR pathway both indicate that this drug may have activity in the microenvironment. In particular, gefitinib may have an effect in the lymph node microenvironments where BCR signaling occurs ${ }^{27}$ and ZAP-70 expression is upregulated. ${ }^{28}$ It is important to note that the complexity of feedback loops and interactions of ZAP-70 in CLL cells are not clearly understood, making it difficult to definitively determine the precise action of gefitinib. This will be the focus of future investigations.

Despite inefficient tyrosine kinase activity in CLL, ${ }^{29}$ ZAP-70 still plays an important role in the overactivation of the BCR pathway. Although the kinase domain is not required for enhanced signaling, inhibition of its kinase activity may cause steric hindrance or prevent conformational changes of signaling complexes preventing downstream signaling events.

Overall, gefitinib selectively targets CLL cells expressing ZAP-70. This indicates that tyrosine kinase inhibitors could be used to selectively treat patients with high ZAP-70-expressing CLL cells. As gefitinib is already in clinical use in lung cancer patients, and lacks suppression of the bone marrow or immune system, further studies are warranted to investigate the clinical activity of gefitinib in ZAP-70+ CLL patients.

\section{Materials and Methods}

Cell isolation and culture. Peripheral blood samples were collected from patients following informed consent in accordance with the Research Ethics Board at the University of Manitoba. Samples were mixed with RosetteSep (Stemcell Technologies, Vancouver, BC, Canada) if the lymphocyte count was $<40 \times 10^{9} /$ and then purified on a Ficoll-Paque gradient (GE Healthcare, Cleveland, OH, USA). Red blood cells (RBCs) were lysed with a RBC lysis buffer (eBioscience, San Diego, CA, USA). All blood samples were processed within $24 \mathrm{~h}$ after collection and used 
fresh. For experiments, the leukemia cells were grown in Hybridoma serum-free medium (SFM, Life Technologies, Carlsbad, CA, USA).

BJAB (ATCC, Burlington, ON, Canada), NALM6 (DSMZ, Braunschweig, Germany), 183 (kind gift from Dr. Panasci, McGill University, Montreal, Canada), Jurkat (ATCC), and Raji+/ - ZAP-70 (kind gifts from Dr. Marshall, University of Manitoba, Winnipeg, MB, Canada) cell lines were all cultured in Hyclone RPMI with 10\% Hyclone fetal bovine serum (FBS; Thermo Fisher Scientific, Waltham, MA, USA).

Drugs and stimuli. Gefitinib (LC Laboratories, Woburn, MA, USA), erlotinib (LC Laboratories), dasatinib (LC Laboratories), ibrutinib (SelleckBio, Houston, TX, USA), and fludarabine (Sigma, St. Louis, MO, USA) were all dissolved in DMSO (Thermo Fisher Scientific) and added. B cells were stimulated with $10 \mu \mathrm{g} / \mathrm{ml}$ biotinylated $\mathrm{Fab}_{2}{ }^{\prime}$ IgM (Southern Biotech, Birmingham, AL, USA) and T cells were stimulated with $2 \mu \mathrm{g} / \mathrm{ml}$ LEAF-purified anti-CD3 (BioLegend, San Diego, CA, USA). For western blot experiments, $10 \mu \mathrm{g} / \mathrm{ml}$ soluble anti-lgM was added and cells were lysed within $1 \mathrm{~h}$. For cell death analysis after $24 \mathrm{~h}, 0.1 \mu \mathrm{g}$ anti-lgM was immobilized in Hanks' buffered salt solution (HBSS, Life Technologies) in a 96-well Falcon plate (BD, Franklin Lakes, NJ, USA) overnight, and then washed before addition of CLL cells.

Cell viability assays. For MTT assays, $3 \times 10^{7} \mathrm{CLL}$ cells in $3 \mathrm{ml}$ of Hybridoma SFM were added to tubes (Sarstedt, Nümbrecht, Germany) and treated with 1, 2, 5, 10 , or $15 \mu \mathrm{M}$ of the drug for $24 \mathrm{~h}$. Cells were washed in HBSS and seeded into 96-well plates for 3 days. On day 3 , MTT (Sigma) was added to a final concentration of $0.25 \mathrm{mg} / \mathrm{ml}$. Plates were incubated for $5 \mathrm{~h}$ at $37^{\circ} \mathrm{C}$ with $5 \% \mathrm{CO}_{2}$, and absorbance was measured at a wavelength of $540 \mathrm{~nm}$.

For flow cytometry, samples were collected, washed with $1 \times$ annexin $V$ Binding Buffer (BD Biosciences, Franklin Lakes, NJ, USA), and then stained with 7AAD (BD) and annexin V-fluorescein isothiocyanate (FITC; BD) or annexin V-allophycocyanin (APC; BD). Transduced Raji cells were stained instead with propidium iodide (PI) or annexin V-APC (BD) because of the GFP vector. Samples were examined using a BD FACSCalibur.

Western blotting and immunoprecipitation. Cell lysates were collected at the indicated times in $1 \% \mathrm{NP}-40$ lysis buffer with complete protease inhibitor tablet (Roche, Basel, Switzerland), $1 \mathrm{mM}$ phenylmethanesulfonylfluoride (PMSF), and $2 \mathrm{mM}$ sodium orthovanadate (New England BioLabs, Ipswich, MA, USA). Protein levels were quantified with a Pierce BCA kit (Thermo Fisher Scientific) according to the manufacturer's instructions. Samples were run on $8-10 \%$ polyacrylamide gels and transferred onto nitrocellulose membranes (Bio-Rad, Hercules, CA, USA) blocked in 5\% BSA (Sigma) or milk in TBS-T as per the antibody manufacturer's suggestions. Primary antibodies included rabbit or mouse anti-ZAP-70 (Cell Signaling, Beverly, MA, USA), rabbit anti-Syk/ZAP-70-P (Cell Signaling), rabbit anti-Lyn (Cell Signaling), rabbit anti-Lyn-P (Epitomics, Burlingame, CA, USA), mouse anti-Lck (Cell Signaling), rabbit anti-Btk (Cell Signaling), rabbit anti-Btk-P (Cell Signaling), rabbit anti-ERK1/2 (Cell Signaling), rabbit or mouse antiERK1/2-P (Cell Signaling), rabbit anti-Akt (Cell Signaling), rabbit anti-Akt Ser-P (Cell Signaling), mouse anti-tyrosine-P (Millipore, Darmstadt, Germany), rabbit anti-Mcl-1 (Cell Signaling), rabbit anti-PARP (Cell Signaling), rabbit anti-cleaved caspase 3 (Cell Signaling), mouse anti-glyceraldehyde-3-phosphate dehydrogenase (antiGAPDH; Sigma), rabbit anti- $\alpha$-tubulin (Cell Signaling), and rabbit or mouse anti- $\beta$ actin (Sigma). Secondary antibodies were goat anti-rabbit-HRP or anti-mouse-HRP (Bio-Rad). Detection of protein was with Pierce ECL or Pierce Supersignal Pico (Thermo Fisher Scientific) reagents. Co-immunoprecipitation was carried out with $500 \mu \mathrm{g}$ of protein in $0.2 \%$ CHAPS lysis buffer containing $150 \mathrm{mM} \mathrm{NaCl}, 20 \mathrm{mM}$ Tris, $10 \%$ glycerol, $2 \mathrm{mM}$ EDTA, $1 \mathrm{mM}$ PMSF, $2 \mathrm{mM}$ sodium orthovanadate, and complete protease inhibitor tablet (Roche). Lysates were incubated at $4^{\circ} \mathrm{C}$ with primary antibody mouse anti-Syk (Abcam, Cambridge, UK) or mouse anti-ZAP-70 (Cell Signaling) on a rotator overnight. Pierce protein $G$ plus agarose bead slurry (Thermo Fisher Scientific) was added in a final dilution of $1: 10$ for $2 \mathrm{~h}$ and the procedure for western blot was followed. Entire immunoprecipitate supernatant was loaded on to a gel lane. Immunoprecipitations were done in the same way, with the exception of using $1 \%$ NP-40 lysis buffer.

Flow cytometry. For extracellular staining of $T$ and $B$ cells, peripheral mononuclear cells were stained with annexin V-FITC, anti-CD3-PE, 7AAD, and antiCD19-APC (BD Biosciences). Cells gated on either CD19+ or CD3+ were then analyzed for expressions of annexin $\mathrm{V}$ and $7 \mathrm{AAD}$.
For intracellular staining of ZAP-70 in cell death experiments, RosetteSep and ficoll-purified CLL cells were first surfaced stained with annexin V-APC and 7AAD, then fixed with solution A (Beckman Coulter, Brea, CA, USA) for 12 min at $37^{\circ} \mathrm{C}$, washed with PBS, permeabilized with solution B (Beckman Coulter) for 5 min at room temperature, and then stained with mouse anti-human ZAP-70-FITC (Beckman Coulter) for $15 \mathrm{~min}$. Mouse IgG1-FITC (BD) was used as an isotype control. Unstained and single-stained controls were always included in all flow cytometry experiments. All sample data were acquired on BD FACSCalibur and analyzed using CellQuest Pro software (BD). CLL cell samples were considered ZAP- $70+$ if $\geq 20 \%$ of the cells stained positively.

The ZAP-70 status of each patient sample was determined by diagnostic flow cytometry. Whole blood was stained with anti-CD19, anti-CD5, anti-CD38, and anti-ZAP-70. The negative control is a normal blood sample that should not have CD19+CD5+ leukemic cells. The positive control is the autologous CD19-CD5+ ZAP-70+ T cells.

Transduction of ZAP-70. Vectors encoding GFP pWPTS or vector encoding ZAP-70 pWPTS-ZAP-70 were packaged into lentivirus by co-transfection with pCMV-R8.91 and pMD.G into HEK-293 T cells. ${ }^{30,31}$ All vectors were kindly provided by Dr. Ferenc Boldizsar (University of Pécs, Pécs, Hungary). Raji cells (originally from DSMZ) were transduced with lentiviral particles using a spin protocol as previously described. ${ }^{32,33}$ Expression of ZAP-70 was confirmed by western blot and flow cytometry.

Statistical analysis. Graphs were created and statistics were performed using GraphPad Prism4 software (GraphPad Software Inc., San Diego, CA, USA). Unless otherwise noted, a paired or unpaired two-tailed $t$-test was performed according to the nature of data. Statistical significance was noted in the figures as ${ }^{*} P<0.05$, ${ }^{* *} P<0.01$, or ${ }^{* *} P<0.001$. Densitometry was calculated using ImageJ (Wayne Rasband; National Institute of Mental Health, Bethesda, MD, USA).

\section{Conflict of Interest}

The authors declare no conflict of interest.

Acknowledgements. We thank the Manitoba CLL Tumor Bank for organization of patient samples, Michelle Brown for the mutational analysis of patient samples, and Dr. Aaron Marshall and Hongzhao Li for the transduced Raji cell lines. This research is supported by the CancerCare Manitoba Foundation operating grant and by CLL Global Research Foundation.

\section{Author contributions}

RFD, WX, J-YY, and EN designed and performed experiments. RD and SBG wrote the paper. $\mathrm{HL}$ and AJM provided vital reagents. VB, JBJ, and SBG provided intellectual input and editorial advice.

1. Shanafelt TD, Kay NE. The clinical and biologic importance of neovascularization and angiogenic signaling pathways in chronic lymphocytic leukemia. Semin Oncol 2006; 33 : 174-185.

2. Hamblin TJ, Davis Z, Gardiner A, Oscier DG, Stevenson FK. Unmutated Ig V(H) genes are associated with a more aggressive form of chronic lymphocytic leukemia. Blood 1999; 94 : 1848-1854.

3. Rassenti LZ, Jain S, Keating MJ, Wierda WG, Grever MR, Byrd JC et al. Relative value of ZAP-70, CD38, and immunoglobulin mutation status in predicting aggressive disease in chronic lymphocytic leukemia. Blood 2008; 112: 1923-1930.

4. Gobessi S, Laurenti L, Longo PG, Sica S, Leone G, Efremov DG. ZAP-70 enhances B-cell-receptor signaling despite absent or inefficient tyrosine kinase activation in chronic lymphocytic leukemia and lymphoma B cells. Blood 2007; 109: 2032-2039.

5. Quiroga MP, Balakrishnan K, Kurtova AV, Sivina M, Keating MJ, Wierda WG et al. B-cell antigen receptor signaling enhances chronic lymphocytic leukemia cell migration and survival: specific targeting with a novel spleen tyrosine kinase inhibitor, R406. Blood 2009; 114: 1029-1037.

6. Lopez-Guerra M, Xargay-Torrent S, Perez-Galan P, Saborit-Villarroya I, Rosich L, Villamor N et al. Sorafenib targets BCR kinases and blocks migratory and microenvironmental survival signals in CLL cells. Leukemia 2012; 26: 1429-1432.

7. Lopez-Guerra M, Roue G, Perez-Galan P, Alonso R, Villamor N, Montserrat E et al. p65 activity and ZAP-70 status predict the sensitivity of chronic lymphocytic leukemia cells to the selective IkappaB kinase inhibitor BMS-345541. Clin Cancer Res 2009; 15: 2767-2776. 
8. Campbell L, Blackhall $\mathrm{F}$, Thatcher N. Gefitinib for the treatment of non-small-cell lung cancer Expert Opin Pharmacother 2010; 11: 1343-1357.

9. Swaisland HC, Smith RP, Laight A, Kerr DJ, Ranson M, Wilder-Smith CH et al. Single-dose clinical pharmacokinetic studies of gefitinib. Clin Pharmacokinet 2005; 44: 1165-1177.

10. Brehmer D, Greff Z, Godl K, Blencke S, Kurtenbach A, Weber M et al. Cellular targets of gefitinib. Cancer Res 2005; 65: 379-382.

11. Stegmaier K, Corsello SM, Ross KN, Wong JS, Deangelo DJ, Golub TR. Gefitinib induces myeloid differentiation of acute myeloid leukemia. Blood 2005; 106: 2841-2848.

12. Hahn CK, Berchuck JE, Ross KN, Kakoza RM, Clauser K, Schinzel AC et al. Proteomic and genetic approaches identify Syk as an AML target. Cancer Cell 2009; 16: 281-294.

13. Calpe E, Codony C, Baptista MJ, Abrisqueta P, Carpio C, Purroy N et al. ZAP-70 enhances migration of malignant $B$ lymphocytes toward CCL21 by inducing CCR7 expression via IgMERK1/2 activation. Blood 2011; 118: 4401-4410.

14. Begleiter A, Verburg L, Ashique A, Lee K, Israels LG, Mowat MR et al. Comparison of antitumor activities of 2-chlorodeoxyadenosine and 9-beta-arabinosyl-2-fluoroadenine in chronic lymphocytic leukemia and marrow cells in vitro. Leukemia 1995; 9: 1875-1881.

15. Amrein PC. The potential for dasatinib in treating chronic lymphocytic leukemia, acute myeloid leukemia, and myeloproliferative neoplasms. Leuk Lymphoma 2011; 52: 754-763.

16. Buchner M, Fuchs S, Prinz G, Pfeifer D, Bartholome K, Burger M et al. Spleen tyrosine kinase is overexpressed and represents a potential therapeutic target in chronic lymphocytic leukemia. Cancer Res 2009; 69: 5424-5432.

17. Friedberg JW, Sharman J, Sweetenham J, Johnston PB, Vose JM, Lacasce A et al. Inhibition of Syk with fostamatinib disodium has significant clinical activity in non-Hodgkin lymphoma and chronic lymphocytic leukemia. Blood 2010; 115: 2578-2585

18. Hoellenriegel J, Meadows SA, Sivina M, Wierda WG, Kantarjian $\mathrm{H}$, Keating MJ et at. The phosphoinositide 3'-kinase delta inhibitor, CAL-101, inhibits B-cell recepto signaling and chemokine networks in chronic lymphocytic leukemia. Blood 2011; 118 : 3603-3612.

19. Advani RH, Buggy JJ, Sharman JP, Smith SM, Boyd TE, Grant B et al. Bruton tyrosine kinase inhibitor ibrutinib (PCl-32765) has significant activity in patients with relapsed/ refractory B-cell malignancies. J Clin Oncol 2013; 31: 88-94.

20. Rooij MF, Kuil A, Geest CR, Eldering E, Chang BY, Buggy JJ et al. The clinically active BTK inhibitor PCl-32765 targets B-cell receptor- and chemokine-controlled adhesion and migration in chronic lymphocytic leukemia. Blood 2012; 119: 2590-2594.

21. Haura EB, Sommers E, Song L, Chiappori A, Becker A. A pilot study of preoperative gefitinib for early-stage lung cancer to assess intratumor drug concentration and pathways mediating primary resistance. J Thorac Oncol 2010; 5: 1806-1814.

22. McKillop D, Partridge EA, Kemp JV, Spence MP, Kendrew J, Barnett $S$ et al. Tumo penetration of gefitinib (Iressa), an epidermal growth factor receptor tyrosine kinase inhibitor. Mol Cancer Ther 2005; 4: 641-649.

23. Deglesne PA, Chevallier N, Letestu R, Baran-Marszak F, Beitar T, Salanoubat C et al. Survival response to $\mathrm{B}$-cell receptor ligation is restricted to progressive chronic lymphocytic leukemia cells irrespective of Zap70 expression. Cancer Res 2006; 66: 7158-7166.
24. Gobessi S, Laurenti L, Longo PG, Sica S, Leone G, Efremov DG. ZAP-70 enhances B-cell receptor signaling in spite of absent or inefficient tyrosine kinase activation in chronic lymphocytic leukemia and lymphoma B-cells. Blood 2006; 109: 2032-2039.

25. Lopez-Guerra M, Xargay-Torrent S, Perez-Galan P, Saborit-Villarroya I, Rosich L, Villamor N et al. Sorafenib targets BCR kinases and blocks migratory and microenvironmental survival signals in CLL cells. Leukemia 2011; 26: 1429-1432.

26. Veldurthy A, Patz M, Hagist S, Pallasch $\mathrm{CP}$, Wendtner $\mathrm{CM}$, Hallek $\mathrm{M}$ et al. The kinase inhibitor dasatinib induces apoptosis in chronic lymphocytic leukemia cells in vitro with preference for a subgroup of patients with unmutated IgVH genes. Blood 2008; 112: 1443-1452.

27. Herishanu Y, Perez-Galan P, Liu D, Biancotto A, Pittaluga S, Vire B et al. The lymph node microenvironment promotes B-cell receptor signaling, NF-kappaB activation, and tumor proliferation in chronic lymphocytic leukemia. Blood 2011; 117: 563-574.

28. Nolz JC, Tschumpe RC, Pittner BT, Darce JR, Kay NE, Jelinek DF. ZAP-70 is expressed by a subset of normal human B-lymphocytes displaying an activated phenotype. Leukemia 2005; 19: 1018-1024

29. Chen L, Huynh L, Apgar J, Tang L, Rassenti L, Weiss A et al. ZAP-70 enhances IgM signaling independent of its kinase activity in chronic lymphocytic leukemia. Blood 2008; 111: 2685-2692.

30. Kung SK, An DS, Chen IS. A murine leukemia virus (MuLV) long terminal repeat derived from rhesus macaques in the context of a lentivirus vector and MuLV gag sequence results in highlevel gene expression in human T lymphocytes. J Virol 2000; 74: 3668-3681.

31. Szabo M, Czompoly T, Kvell K, Talaber G, Bartis D, Nemeth P et al. Fine-tuning of proximal TCR signaling by ZAP-70 tyrosine residues in Jurkat cells. Int Immunol 2012; 24: 79-87.

32. Costantini JL, Cheung SM, Hou S, Li H, Kung SK, Johnston JB et al. TAPP2 links phosphoinositide 3-kinase signaling to B-cell adhesion through interaction with the cytoskeletal protein utrophin: expression of a novel cell adhesion-promoting complex in B-cell leukemia. Blood 2009; 114: 4703-4712.

33. Tran J, Kung SK. Lentiviral vectors mediate stable and efficient gene delivery into primary murine natural killer cells. Mol Ther 2007; 15: 1331-1339.

(i) Cell Death and Disease is an open-access journal published by Nature Publishing Group. This work is licensed under a Creative Commons Attribution 4.0 International Licence. The images or other third party material in this article are included in the article's Creative Commons licence, unless indicated otherwise in the credit line; if the material is not included under the Creative Commons licence, users will need to obtain permission from the licence holder to reproduce the material. To view a copy of this licence, visit http://creativecommons.org/licenses/by/4.0 